\title{
Automatic Road Extraction based on Normalized Cuts and Level Set Methods
}

\author{
M.Rajeswari \\ Department of \\ Telecommunication \\ Engineering \\ Bangalore Institute of \\ Technology \\ Bangalore560 $004 \mathrm{KA}$, India
}

\author{
K.S.Gurumurthy \\ Department of Electronics \& \\ Communication Engineering \\ University College of \\ Engineering \\ Bangalore $560001 \mathrm{KA}$,India \\ L.Pratap Reddy \\ Department of Electronics \& \\ Communication Engineering \\ JNTU College of Engineering- \\ Kukatpally, \\ Hyderabad-500 085 AP, India
}

\author{
S.N.Omkar \\ Senthilnath.J \\ Department of Aerospace \\ Engineering, Indian Institute \\ of Science \\ Bangalore, 560 012, \\ $\mathrm{KA}$, India
}

\begin{abstract}
Automatic road network extraction based on high resolution satellite image for urban planning holds great potential for significant reduction of database development/updating cost and turnaround time. Satellite remote sensing has been recognized worldwide as an effective technology for the monitoring and mapping the urban development. Two approaches for road network extraction for an urban region have been proposed. When an image is considered in original form it is difficult and computationally expensive to extract roads due to presence of other road-like features with straight edges. Hence roads are first extracted as elongated regions by removing bright regions (that mostly represent the buildings, parking lots and other open spaces), non-linear noise segments are removed median filtering (based upon the fact that road networks constitute large number of small linear structures). The roads are then modeled as boundaries and are extracted using Level set and Normalized cuts methods .Finally The extracted roads are overlayed on the original image. The experimental results show that these approaches are efficient in extracting road segments in urban region from high resolution satellite images. Evaluation of the results carried out by comparing the level set and normalized cuts results with manually extracted reference data. The methods were applied on the high resolution IKONOS image of urban area of Hobart, Australia.
\end{abstract}

\section{Keywords}

Level set, median filtering; Normalized cuts; Performance Evaluation; Urban Road extraction;

\section{INTRODUCTION}

Road extraction from remotely sensed images has always been a challenging problem. Fully automatic extraction of roads from satellite imagery eliminates the need for human operators to perform the time consuming and expensive process of mapping roads from photographs. As increasing volumes of high spatial resolution satellite imagery (e.g., Ikonos, QuickBird, OrbView-3, etc.) become available there is a need for automation to extract information and analyze image content.

Automatic detection and extraction of roads from remotely sensed imagery has been an active research and development topic for the last twenty years and in the practice for the last twelve years, since the $1 \mathrm{~m}$ resolution world's first commercial remote sensing satellite (Ikonos) become available in 1999. Until now, various road extraction methods have been proposed. For rural areas Zhang, C., 2004 [1] have done database verification and updating determining the region of interest for roads by a multispectral classification and excluding high regions using Digital Surface Models then parallel edges are extracted in the regions of interest. Mena, J.B. and Malpica, J.A., 2005 [2] have used three different classification methods for color and texture and are combined to extract road regions. Roads are only extracted in the regions around database roads. Road extraction is difficult in the presence of context objects such as buildings or trees close to the road, disrupting the appearance of the road or occluding it. Gerke.M and Heipke.C 2008[3] and Baumgartner A.et al., [4] have considered context objects. For urban areas Zhang Q and Couloigner I 2006[5] have developed their extraction based on multispectral classification and filtering using shape criteria. Digital surface model (DSM) from LIDAR is used as an additional data source by Hu.X et al., 2004 [6] to restrict the search space for the straight lines. This cannot handle curved roads well. In region based extraction Doucette, $\mathrm{P}$ et al., 2001 [7] use hyper spectral data channels to extract road regions and road pixels are grouped into a network with a kmedian classification. $\mathrm{Hu}, \mathrm{J}$ et al., 2007 [8] extract road footprints based on their shape and then track them. Junction footprints are distinguished from ordinary road footprints. They have used Post-processing to remove false extractions. Bacher, U. and Mayer, H [9] calculates value for the road class pixel then road hypotheses is determined using fuzzy logic. Road network is generated using weighted graph and detour factor to close small and large gaps respectively. Poullis, C. and You, S., 2010 [10] have extracted road using Gabor filter for image classification into road and non-road pixels segmentation using graph cut and Gabor filter for post processing Asef Zare [11]use feature extraction (preprocessing), neuro-fuzzy system modeling and post processing.

Recent methods for extraction of roads from high resolution imagery include Normalized cut and Level set segmentation methods. Level set method is a search algorithm that determines evolving curve's boundary pixels the level set propagates as long as the speed function is greater than zero .For the road extraction problem speed function has to be greater than zero, at the edges of the true road boundary. Therefore Level set is an efficient technique for extracting road. Normalized Cuts is a graph-based method taking both local and global characteristics of the image. Local characteristics are incorporated into the similarity matrix for considering the similarity of pixels in a close neighborhood. Global characteristics are used for computing the best cut. The combination of local and global aspects ignores noise, small surface changes and weak edges and producing extraction with most segments covering only a road area. This makes normalized cuts suitable for automatic road extraction.

In the literature on level set algorithm and normalized Cut for road extraction. Rajeshwari, M et al., [12],Omkar et al.,[13] 
have used Pre-processing for removing noise in the image and then Level Set Evolution without Re-initialization (LSER) with the initial seed point provided by the user. Trish Keaton and Jeffrey Brokish (2002) [14] provide an initial seed point on the road of interest, then evolving the region using level set method. Iterative smoothing is used to refine the extracted region to accurately estimate the road centerline. Fast marching level set method machine learning for parameter tuning and information fusion for refinement of object delineation has been used by X. Cai et al., [15]. M. Ravanbaksh et al., [16] provide junction outline to focus the attention on a specific area later constraints are introduced to distinguish road junctions variational level set method. Bibo $\mathrm{Lu}$ [17] applies Level set for the segmenting sensing image. Then second order moment is calculated and the road networks are extracted using prior information about geometric shape. Normalized cuts is used for initial segmentation step by Qihui Zhu; Mordohai, P. [18] also requires priori information like depth and intensity measurements from the range sensor for LIDAR data and then extracts roads using hypothesis testing. A.Groth et al. [19, 20] have applied Normalized Cuts algorithm for dividing image into Segments with color and edge criteria. Then, the initial segments are grouped to form larger segments and are then evaluated using shape criteria to extract road parts. We propose an approach for road network extraction in urban areas without the priori information about road or DSM. Main goal of the paper is to show the effectiveness of the approaches for Road extraction. Initially image is preprocessed to remove small linear structures appearing as noise then the level set and Normalized cuts are used to extract the roads $\&$ are fully automatic. The quality measures evaluated show that these approaches are better compared to the earlier works based on these methods.

In Section 2 overview of the two methods is explained. In Section 3 description of the road network extraction process is presented in section 4 Experimental results and analysis of qualitative measures are described. Section 5 gives conclusions and future work.

\section{Methodology}

As mentioned above there are various methods used for road extraction. The level set and normalized cuts have an edge over other methods. Level set is a powerful and efficient technique for performing feature extraction by taking the road extraction as a boundary evolution problem. Level set method is used to derive a global road segment

The normalized cuts algorithm defines the optimal Road extraction in terms of an extraction that divides the image into two parts. To achieve a full extraction, an optimal binary extraction is determined and then recursively an optimal extraction within each extraction is achieved.

\subsection{Level set Method}

The level set method (LSM) tracks the evolution of a boundary front which is moving with a speed function that is normal to the boundary curve. In image segmentation [21], active contours are dynamic curves that move toward the object boundaries.

The road extraction problem is considered as a boundary evolution problem within the level set framework. The level set will continue to propagate as long as the speed function is greater than zero. Therefore, the speed function must be properly designed such that as the boundary approaches the desired location. For the road extraction problem, it is desirable for speed function to be greater than zero, at the edges of the true road boundary.
For this an external energy is defined that moves the zero level curve toward the object boundaries [22]. The edge indicator function ' $\mathrm{g}$ ' is defined by:

$$
g(x, y)=\left.\frac{1}{1+\left|\nabla G_{\sigma} * I(x, y)\right|}\right|^{2}
$$

Where ' $\mathrm{I}$ ' is an image, ' $\mathrm{G}_{\sigma}$ ' is the Gaussian kernel with standard deviation ' $\sigma$ '. Then the external energy for a function $\phi(\mathrm{x}, \mathrm{y})$ is given by:

$$
\varepsilon_{\mathrm{g}, \lambda, \mathrm{v}}(\phi)=\lambda \mathrm{L}_{\mathrm{g}}(\phi)+\mathrm{vA}_{\mathrm{g}}(\phi)
$$

Where $\lambda>0$ and $v$ are constants, and the terms $\mathrm{L}_{\mathrm{g}}(\phi)$ and $\mathrm{A}_{\mathrm{g}}(\phi)$ are defined by

$$
\mathrm{L}_{\mathrm{g}}(\phi)=\int_{\Omega} \mathrm{g} \delta(\phi)|\nabla \phi| \mathrm{dxdy}
$$

And

$$
A_{g}(\phi)=\int_{\Omega} g H(-\phi) d x d y
$$

respectively, where $\delta$ is the univariate Dirac function and $\mathrm{H}$ is the Heaviside function. Then total energy function is given by

$$
\varepsilon(\phi)=\mu \mathrm{P}(\phi)+\varepsilon_{\mathrm{g}, \lambda, v}(\phi)
$$

The external energy $E_{g, \lambda, v}$ drives the zero Level Set toward the object boundaries, while the internal energy $\mu \mathrm{P}(\phi)$ penalizes the deviation of $\phi$ from a signed distance function during its evolution. By calculus of variations, the Gateaux derivative (first variation) of the functional Equation (5) can be written as

$$
\begin{aligned}
& \frac{\partial \varepsilon}{\partial t}=-\mu\left[\Delta \phi-\operatorname{div}\left(\frac{\nabla \phi}{|\nabla \phi|}\right)\right] \\
& -\lambda \partial(\phi) \operatorname{div}\left(g \frac{\nabla \phi}{|\nabla \phi|}\right)-v g \partial(\phi)
\end{aligned}
$$

Where $\Delta$ is the Laplacian operator. The function $\phi$ that minimizes this function satisfies the Euler-Lagrange equation $\partial \mathrm{E} / \partial \phi=0$. The steepest descent process for minimization of the functional $\mathrm{E}$ is the following gradient flow:

$$
\frac{\partial \phi}{\partial \mathrm{t}}=\mu\left[\Delta \phi-\operatorname{div}\left(\frac{\nabla \phi}{|\nabla \phi|}\right)\right]+\lambda \partial(\phi) \operatorname{div}\left(\mathrm{g} \frac{\nabla \phi}{|\nabla \phi|}\right)+\operatorname{vg} \partial(\phi)
$$

This gradient flow is the evolution equation of the level set function The second and the third term in the right hand side of Equation (7) correspond to the gradient flows of the energy functional $\lambda \mathrm{L}_{\mathrm{g}}(\phi)$ and $\nu \mathrm{A}_{\mathrm{g}}(\phi)$, respectively, and drive the zero level curve towards the object boundaries.

\subsection{Normalized cuts Method}

In Normalized cut method for extracting road segments, each individual edge weight is a measure for the similarity between two connected pixels. If the road segments that are to be extracted have distinctly different intensities then intensity is used as similarity measure. Multiple similarity measures are combined, since the differentiation cannot be realized using only one criterion. The weight is the combination of all similarity measures, has a value between 0 and 1 . The graph representing the image is then cut into segments aiming at a 
large dissimilarity between different segments and at the same time a large similarity within the segments. Considering an undirected graph $\mathrm{G}=(\mathrm{V}, \mathrm{E})$, where $\mathrm{V}$ is the set of nodes and $\mathrm{E}$ is the set of edges. A pair of nodes $\mathrm{p}$ and $\mathrm{q}$ is connected by an edge and is weighted by $w(p, q)$. Let $\mathrm{X}$ and $\mathrm{Y}$ be a partition of the graph where $\mathrm{X} \cup \mathrm{Y}=\mathrm{V}$ and $\mathrm{X} \cap \mathrm{Y}=\phi$. Therefore cost of cut is defined as the similarity between the groups $\mathrm{X}$ and $\mathrm{Y}$ and it is formulated as:

$$
\operatorname{Cut}(X, \quad Y)=\sum_{p \in X, q \in Y} w(p, q)
$$

Minimum cut is the cut of minimum weight, where weight of cut $(\mathrm{X}, \mathrm{Y})$ is given by equation (8). The limitation of using minimum cut is described in [23.]. The minimum cut usually yields over clustered results when it is recursively applied. This induces several modified graph partition criteria

$$
\operatorname{Ncut}(\mathrm{X}, \mathrm{Y})=\frac{\operatorname{cut}(X, Y)}{\operatorname{volume}(X)}+\frac{\operatorname{cut}(X, Y)}{\operatorname{volume}(Y)}
$$

Where volume $(\mathrm{X})$ is the sum of cost of all edges that touch $\mathrm{X}$ and volume $(\mathrm{Y})$ is the sum of cost of all edges that touch $\mathrm{Y}$ i.e.

$$
\begin{array}{ll}
\operatorname{volume}(\mathrm{X}) & =\sum_{p \in X, q \in V} w(p, q) \\
\operatorname{volume}(\mathrm{Y}) & =\sum_{p \in Y, q \in V} w(p, q)
\end{array}
$$

Normalized cut minimizes $\operatorname{Ncut}(\mathrm{X}, \mathrm{Y})$ maximizes a measure of similarity within the sets $\mathrm{X}$ and $\mathrm{Y}$ and this results in optimal partition. If $W$ is the cost matrix, i.e. $W(i, j)=c i, j$; is the weight between the nodes $i$ and $j$ in the graph and $D$ is the diagonal matrix such that $D(i, i)=\sum_{j} W(i, j)$ is the sum of costs from node $\mathrm{i}$ and $\mathrm{D}(\mathrm{i}, \mathrm{j})=0$. Based on this input, the optimal partition can be determined by computing:

$$
\begin{gathered}
\min \operatorname{Ncut}(\mathrm{X}, \mathrm{Y})=\min _{\mathrm{y}} \frac{\mathrm{y}^{\mathrm{T}}(\mathrm{D}-\mathrm{W}) \mathrm{y}}{\mathrm{y}^{\mathrm{T}} \mathrm{Dy}} \\
y(i) \in\{1,-b\}, 0<b \leq 1, \text { and } \mathbf{y}^{T} D 1=0
\end{gathered}
$$

If $y$ is takes real values then the minimization of equation (10) is by solving the generalized eigen value system.

$$
(\mathrm{D}-\mathrm{W}) \mathrm{y}=\lambda \mathrm{Dy}
$$

To compute the cut, graph is divided into two clusters. For good segmentation, the weight on the edges should represent pixels affinity for being in the same group. The weights for the pixel pairs are inserted in a symmetric similarity matrix (W-matrix) whose row and column dimensions equal the number of pixels. The weight between regions $i$ and $j$ is defined as

$$
\begin{gathered}
W=N \times N \text { symmetric matrix, where } \\
\mathrm{W}(\mathrm{i}, \mathrm{j})=\left\{\begin{array}{cc}
\mathrm{e}^{-\left\|\mathrm{F}_{\mathrm{i}}-\mathrm{F}_{\mathrm{j}}\right\| / \sigma_{\mathrm{F}}^{2}} \times \mathrm{e}^{-\left\|\mathrm{X}_{\mathrm{i}}-\mathrm{X}_{\mathrm{j}}\right\| / \sigma_{\mathrm{X}}^{2}} & \text { if } \mathrm{j} \in \mathrm{N}(\mathrm{i}) \\
0 & \text { otherwise }
\end{array}\right. \\
\left\|F_{i}-F_{j}\right\|=\text { Image feature similarity } \\
\left\|X_{i}-X_{j}\right\|=\text { Spatial Proximity }
\end{gathered}
$$

$$
\begin{gathered}
D=N \times N \text { diagonal matrix, where } \\
D(i, i)=\sum_{j} W(i, j)
\end{gathered}
$$

Larger weight edges for pixels have similar intensity are close to each other. The minimum is determined by calculating the eigenvectors of a matrix derived from the $\mathrm{W}$-matrix using equation (12). The "generalized" eigen system in (11) is transformed into a "standard" eigen value problem as

$$
\begin{aligned}
& D^{-\frac{1}{2}}(D-W) D^{-\frac{1}{2}} Z=\lambda Z \\
& \text { where } Z=D^{\frac{1}{2}} y
\end{aligned}
$$

The road extraction process includes pre-processing, segmentation and the image overlaying.

\subsection{Pre-processing}

Pre-processing is required to improve the image quality and to generate the elongated road network for further processing. Firstly, the panchromatic image was grouped into clusters in an unsupervised classification. This was followed by a grouping operation to reduce the noise and smoothening of the image. This improves the tolerance as the noise is reduced and hence minimizes the effects of using a high resolution imagery (at such high-resolutions the road is susceptible to noise in the form of vehicular traffic, road markings, structural shadows, occlusions and many road surface contrast irregularities). Filtering operation generates the elongated road regions.

\subsubsection{Classification}

The image used is an IKONOS, $1 \mathrm{~m}$ resolution satellite image of the Hobart area in Tasmania, Australia. It has dimensions of $467 \times 327$ pixels. By setting the threshold value 0.95 , the high resolution image was grouped into 50 clusters in an unsupervised classification, five of which correspond to road networks. A level one classification was carried out by dividing the image into two classes: Roads and non-roads. A lot of misclassifications were noted among roads and barren land.

\subsubsection{Grouping}

A nearest neighbourhood grouping (NNG) operation is applied to the classified representation for smoothening the spectral response within the pixel's local neighbourhood. In this process the vehicular occlusions and small patches of road contrast abnormalities are eliminated. In this method a pixel is chosen. Its surrounding eight neighbours are considered for voting. If a class gets four or more votes, then the chosen pixel is assigned to that class. Otherwise the pixel retains its class. Road pixels in the neighbourhood grow over such noise elements and homogeneous

\subsubsection{Filtering}

The method of road extraction in certain locations poses challenge because the spectral reflectance of some of the old buildings (or buildings with a type of construction that renders a dark road like effect) resembles the road surface. Such 
buildings form the clutter and these non-road structures need to be removed. A morphological opening operation is applied which identifies road blocks. The pattern matching is done focusing on retrieving a road network of specific width. Salient road attribute is its linear structure which can be used for extracting elongated road regions. A linear operator (predefined/user-defined length) controls the filtering of the road segments not belonging to the global road network. For the above resultant image median filtering technique is applied. Median filtering is similar to using an averaging filter, in that each output pixel is set to an average of the pixel values in the neighborhood of the corresponding input pixel. However, with median filtering, the value of an output pixel is determined by the median of the neighborhood pixels, rather than the mean. The median is much less sensitive than the mean to extreme values (called outliers). Median filtering is therefore better able to remove these outliers without reducing the sharpness of the image regions/segments are generated.

\subsection{Segmentation}

Segmentation is the process of partitioning the digital image into multiple segments. Image segmentation is the process of assigning a label to every pixel in an image with pixels having same label share certain visual characteristics. The flow charts of the level set and the normalized cuts are as shown in figure 1 and figure 2

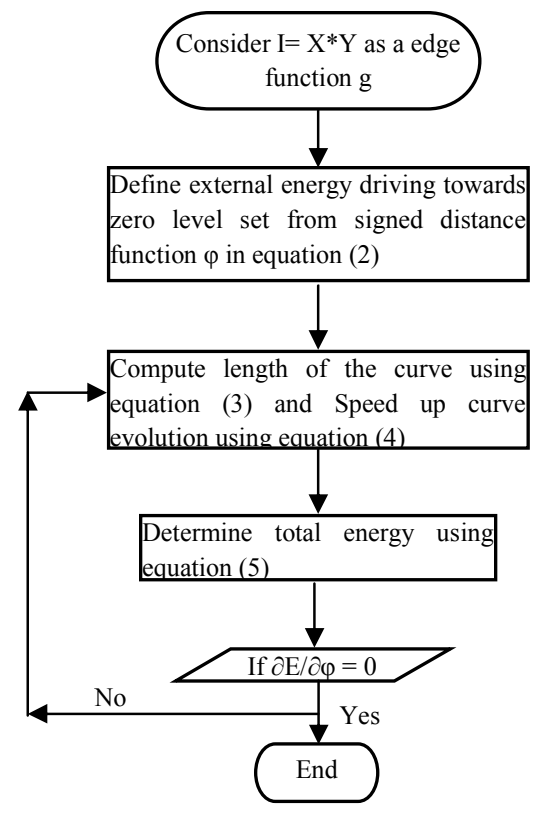

Figure 1 Flow chart for Level set

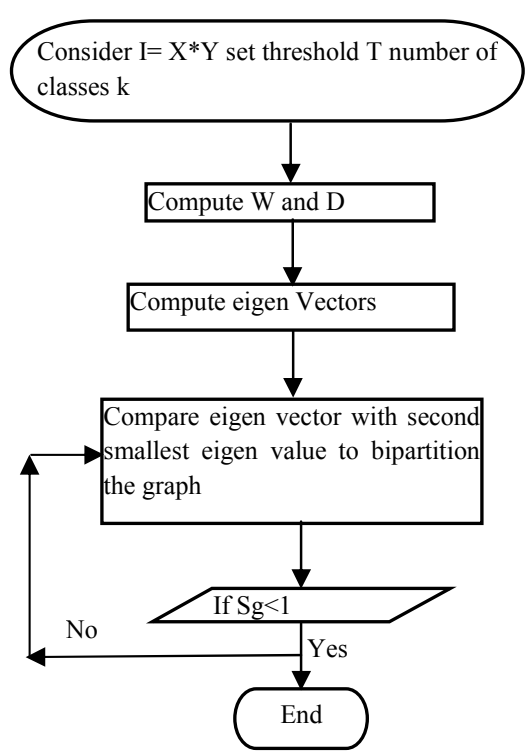

Figure 2 Flow chart for Normalized Cut

\subsection{Image Overlaying}

In order to illustrate the accuracy, the extracted road region using is converted into binary image format. This binary image is overlaid on the original panchromatic image leads to display the road topology by avoiding the complex noise element. In the overlaid image the thin lines indicate the road topology.

\section{Performance evaluation}

The automatically extracted roads are compared with manually traced reference roads using ERDAS to perform accuracy assessment. Because roads are linear features, it is possible to use all the data rather than just sample points to conduct the accuracy assessment Wiedemann et al[24] proposed several quality measures to evaluate the quality of extracted roads. The following are the measures for accuracy assessment of road extraction:

Completeness $=\frac{\mathrm{TP}}{\mathrm{TP}+\mathrm{TN}^{\circ}}$
Correctness $=\frac{\mathrm{TP}}{\mathrm{TP}+\mathrm{FN}}$
Quality $=\frac{\mathrm{TP}}{\mathrm{TP}+\mathrm{FP}+\mathrm{FN}}$
Re dundancy $=\frac{\mathrm{TP}-[1-\mathrm{FP}+\mathrm{FN}]}{\mathrm{TP}}$

RMSE $=\sqrt{\frac{\sum_{i=1}^{N} d\left(\text { der }_{i}-\text { ref }\right)^{2}}{N}}$

$\mathrm{N}=$ number of pieces of unmatched extraction

Number of gaps per $\mathrm{km}=\frac{\mathrm{n}}{\text { Length of reference }[\mathrm{km}]}$ $\mathrm{n}=$ number of gaps 
Mean gap length $[\mathrm{m}]=\frac{\sum_{\mathrm{i}=1}^{\mathrm{n}} \mathrm{g}(\mathrm{li})}{\mathrm{n}}$

$$
l_{i}=\text { length of the } \mathrm{i}^{\text {th }} \text { gap }(\mathrm{m})
$$

Completeness represents the percentage of reference data being correctly extracted. Correctness indicates the percentage of correctly extracted roads. Therefore, completeness is producer's accuracy and Correctness is users' accuracy. The quality represents the overall accuracy Redundancy gives the percentage of correctly extracted roads which overlap as they correspond to the same ideal road RMSE (Root Mean Square error) expresses the geometrical accuracy of extracted roads, the number of gaps per unit length and the mean gap length, where a gap corresponds to a part of the ideal road that is not found.

To calculate these quality measures, buffer zones are built around the extracted roads and the reference roads. The buffer width is considered as approximately half of the actual road width. The direction difference is derived directly from the vector representations of both networks.A true positive (TP) is the obtained result coinciding with the reference result. A false positive (FP) is where there is a road pixel in the obtained result that is not in the reference data. A false negative $(\mathrm{FN})$ is where there is a road pixel in the reference data and not present in the obtained result.

\section{Results and discussion}

The data considered is the IKONOS pan-sharpened multi spectral satellite image from Hobart area in Australia. The image with three bands of one meter ground resolution covering areas of a $467 \times 327$ pixels is considered. The quality measures were calculated using the equations (15)-(21). The images were executed on a system with $1.83 \mathrm{GHz}$ and $2 \mathrm{~GB}$ RAM using Matlab 7.4.

Figure 3 is a 1 meter resolution panchromatic IKONOS image ( $467 \times 327$ pixels). Figure 4 is the classified image of Figure 3 . Figure 5 is the filtered image using median filtering. Figure 6 and 7 are extracted road images using level set and normalized cut methods after overlaying the original and extracted image. Figure 8 and Figure 9 show the comparison of road extraction results of two methods. The pre processed image is given as input to level set algorithm. Earlier road extractions using level set required road seed points and is eliminated in this paper by setting the radius of pixels circle and signed distance to the pixel circle are set to 5 and 2 respectively for the road feature extraction. The result obtained by the level set method is shown in Figure 6 .It is seen that level set method extracts road boundaries accurately in the presence of other objects on the road, surface properties of the road. But the backgrounds of high resolution remote sensing images are quite complex, those objects whose spectral properties similar to the roads have also been extracted, which appear as noise (small irregular shapes) in the extracted image.
The normalized cuts algorithm is applied for the pre processed image to extract the road segments. Earlier road extractions used normalized cut for initial segmentation leading to over segmentation and additional steps are used to remove non road segments. The image is divided into road segments by the Normalized Cuts algorithm. Figure 7 shows that the extraction is successful as most segments cover only a road area. The combination of local and global characteristics is an important aspect of the Normalized Cuts algorithm. It ignores noise, small surface changes and weak edges. Figure 7 has several road segments and also contains a non road segment in top right side of the image. Since the segments are defined by the image content, and other objects have similar radiometric and geometric properties to road parts resulting in false road.

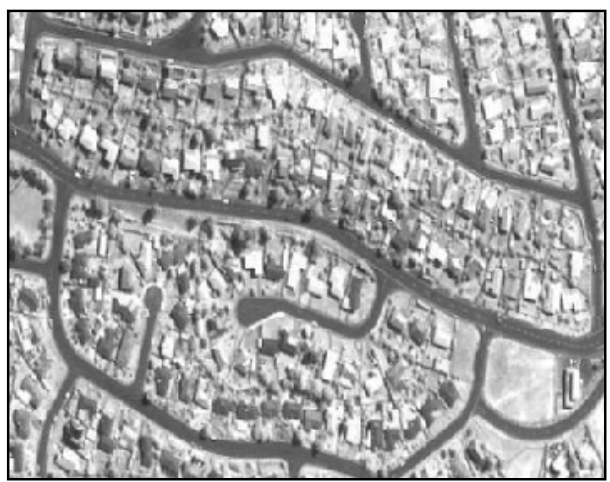

Figure 3: Original Image

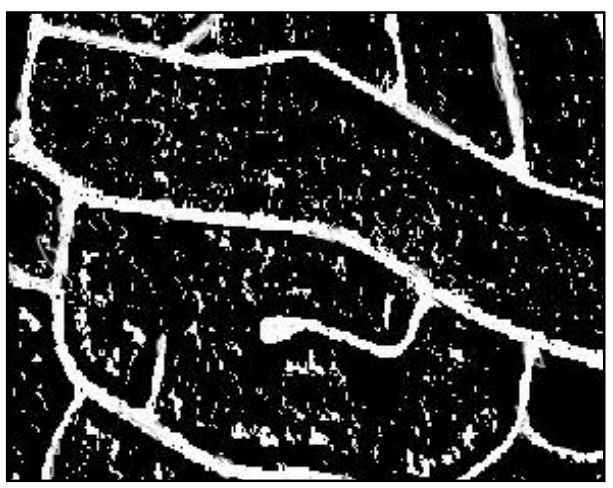

Figure 4: Classified Image

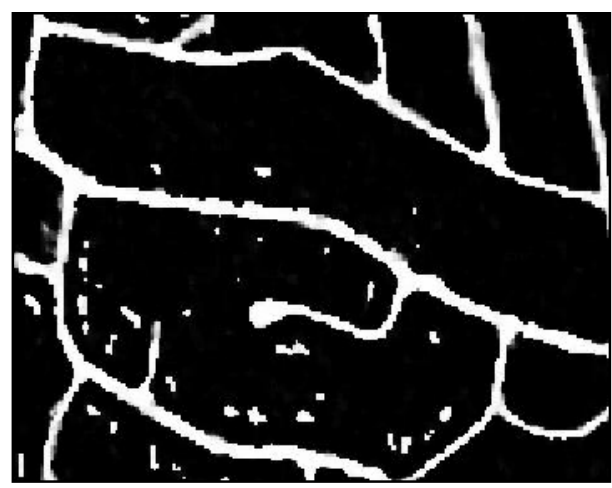

Figure 5: Filtered Image 


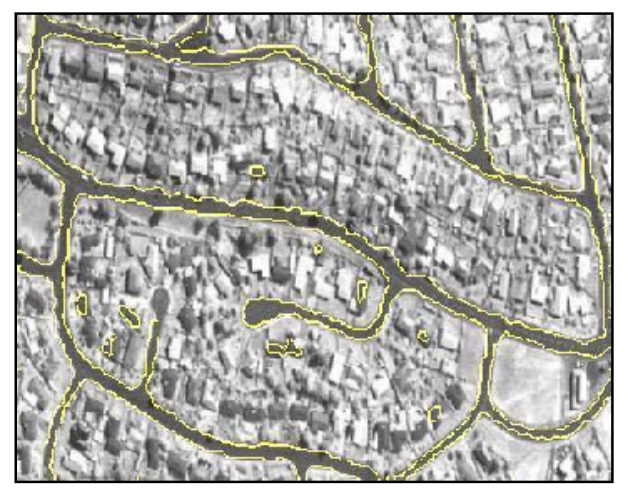

Figure 6: Extracted image using Level set overlayed on Original Image

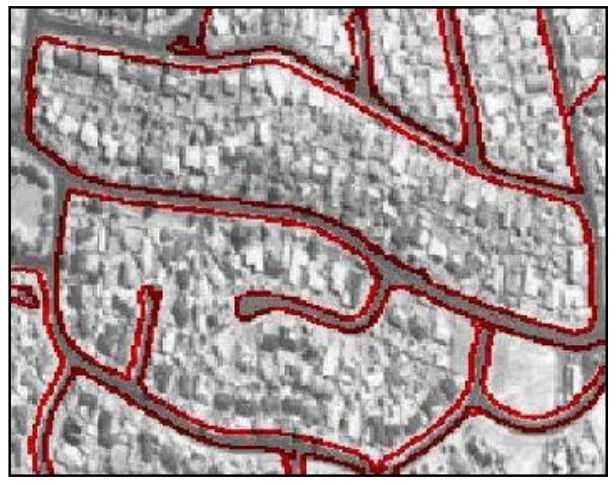

Figure 7: Extracted image using Normalized cuts overlayed on Original Image

To show the effectiveness of the approaches used more clearly the quantitative analysis of the automatically extracted road network is done by comparing them with the manually extracted road regions using ERDAS. The manually extracted regions include areas occluded by shadows or trees. The completeness, correctness, quality, redundancy, RMS and gap statistics of the road parts are computed according to Weidemann et al., [29] the results are shown in figure 8 and figure 9. They are determined on a per-pixel level and thus refer to the extracted areas.

Figure 8 gives the completeness and correctness values for the two methods. Completeness of $95.56 \%$ was obtained using normalized cut compared to $93.9 \%$ using level set method which is still better than that obtained in [16]. The correctness is $87.89 \%$ for level set and $97.25 \%$ for Normalized cuts. The accuracy of normalized cut is $93.15 \%$ compared to $87.3 \%$ in level set. Figure 9 shows that the redundancy is more in level set method (0.158) compared to that in normalized cuts (0.018) and the overall number of gaps is smaller and the mean gap length is more in normalized cuts. RMS reflects the potential accuracy of an automatically segmented region. Normalized cut when compared with [20] also has produced better results. Overall normalized cuts are better than level set method in extracting road network.

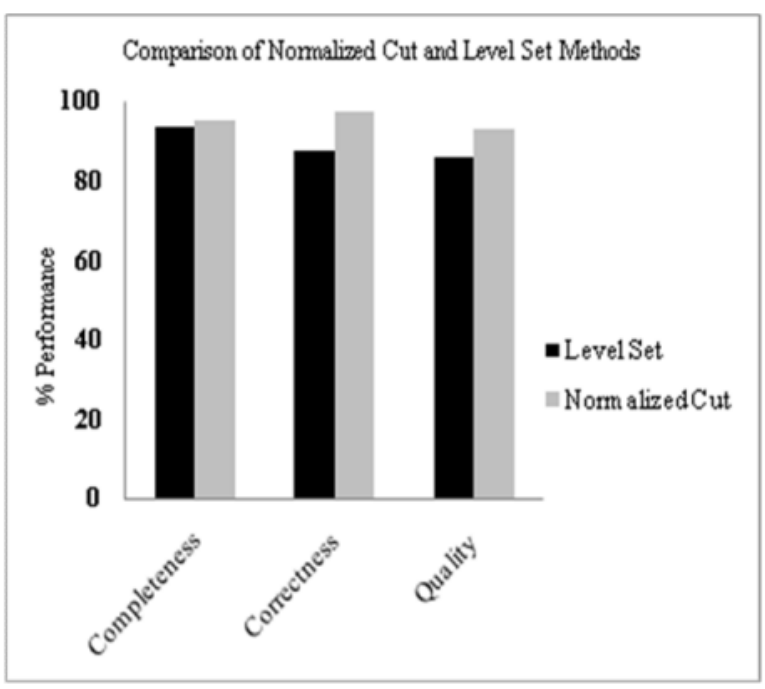

Figure 8: Comparison of Completeness, Correctness and Quality measures

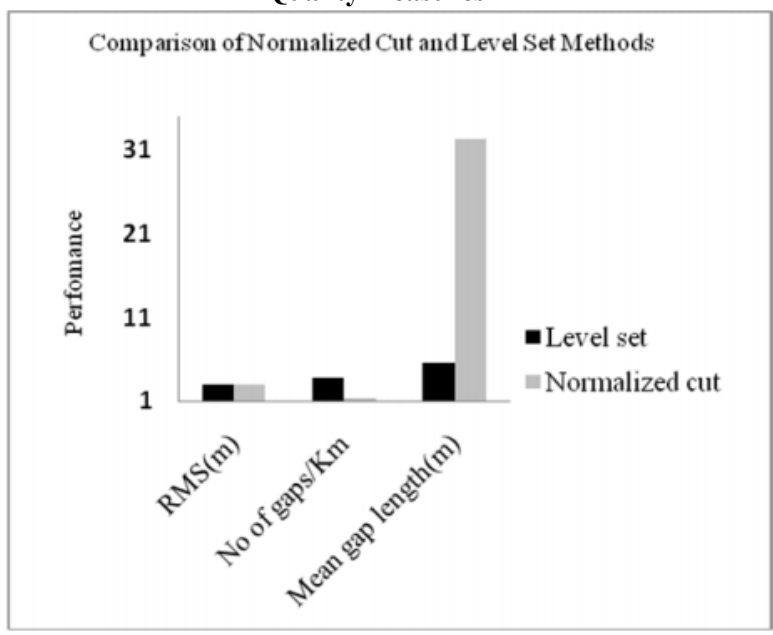

Figure 9: Comparison of RMS (m), No of Gaps/km and Mean gap length measures

\section{CONCLUSIONS}

Automatic Road Network extraction is an important feature in urban planning. Urban planners require methods for fast road data through satellite remote sensing techniques Automatic extraction of road network information has problems, especially in urban areas .To overcome such problems two approaches have been proposed and have shown good results. Normalized cuts have produced results superior to level set method. When compared with literature using the same method level set has also performed well in our approach. The main contribution of this paper is using normalized cut and level set on the preprocessed data to produce greater accuracy. There is further scope for refining the results of the level set method such as removing unexpected road lines generated and extracting unidentified road regions. The limitation of normalized cuts is the huge storage requirement and time complexity when the image size increases and requirement of partitioning into equal segments have to be considered. Future 
work includes addressing these issues to obtain complete accuracy in road extraction.

\section{ACKNOWLEDGMENTS}

This work is supported by the Space Technology Cell, Indian Institute of Science and Indian Space Research Organization grant.

\section{REFERENCES}

[1] Zhang, C., 2004. Towards an operational system for automated updating of road databases by integration of imagery and geodata. ISPRS J. .Photogramm. 58(3-4), 2004 pp. $166-186$

[2] Mena, J.B. and Malpica, J.A., 2005. An automatic method for road extraction in rural and semi-urban areas starting from high resolution satellite imagery. Pattern Recogn. Lett. 26(9), 2005pp.1201-1220.

[3] Gerke, M. and Heipke, C., 2008. Image-based quality assessment of road databases. Int. J. Geogr. Inf. Sci., 22(8), 2008 pp.871-894.

[4] Baumgartner, A., Steger, C., Mayer, H., Eckstein, W. and Ebner, H., 1999. Automatic road extraction based on multiscale, grouping, and context. Photogramm. Eng. Rem. S. 65(7), 1999pp. 777-785

[5] Zhang, Q. and Couloigner, I., 2006. Automated road network extraction from high resolution multi-spectral imagery. In: Proc.ASPRS Annual Conf., Reno, Nevada, $200610 \mathrm{p}$

[6] Hu X, Tao CV and Hu Y (2004) Automatic Road Extraction from dense urban area by integrated processing of high-resolution imagery and lidar data. XXth ISPRS Congress, Istanbul, 12-23 July 2004. (Available online at: http://www.isprs.org/istanbul2004/comm3/papers/288.pd f- International archives of photogrammetry and Remote Sensing, Vol. XXXIII, part B3. Amsterdam

[7] Doucette, P., Agouris, P., Stefanidis, A. and Musavi, M., 2001.Self-organized clustering for road extraction in classifiedimagery. ISPRS J. Photogramm. 55(5-6), 2001 pp. $347-358$

[8] Hu, J., Razdan, A., Femiani, J.C., Cui, M. and Wonka, P., 2007.Road network extraction and intersection detection from aerial images by tracking road footprints. IEEE TGARS 45(12), 2007 pp.4144-4156

[9] Bacher, U. and Mayer, H. Automatic road extraction from multispectral high resolution satellite images. In. Stilla U, Rottensteiner F, Hinz S (Eds) CMRT05. IAPRS, Vol. XXXVI, Part 3/W24, Vienna, Austria. 2005.

[10] Poullis, C. and You, S., 2010. Delineation and geometric modeling of road networks. ISPRS J. Photogramm. 65(2), 2010 pp.165-181.

[11] Asef Zare, Mostafa, OkautiAutomatic road extraction based on neuro-fuzzy algorithm, Proceeding, ROCOM'10 Proceedings of the 10th WSEAS international conference on Robotics, control and manufacturing technologyWorld Scientific and Engineering Academy and Society (WSEAS) Stevens Point, Wisconsin, USA C2010

[12] Rajeshwari, M ,Senthilnath, J.; .; Omkar, S. N.Semi Automatic Road Extraction using high resolution satellite imagery in urban areas, Indian Engineering Congress 2007, Uadipur ,Rajasthan, 14-15 December, 2007

[13] S. N. Omkar, Senthilnath J and M. Rajeswari, "A Variational Level Set method for road extraction in satellite images", in the Proceedings of the Conference on Advances in Space Science and Technology, CASST'S 2008, , IIT Kharagpur. January 14-16, 2008

[14] Trish Keaton and Jeffrey Brokish (2002). A level set method for the extraction of roads from Multispectral Imagery, Proceedings of the 31st Applied Imagery Pattern Recognition Workshop (AIPR.02) 0-7695-1863$\mathrm{X} / 02 \$ 17.00$ (C) 2002 IEEE

[15] X. Cai, A. Sowmya and J. Trinder (2006), Machine Learning Approach for Automatic Road Extraction, Proceedings of the ASPRS 2006 Annual Conference, Reno, Nevada, USA, May 1-5, 2006.

[16] M. Ravanbakhsh, C. Heipke, K. Pakzad ,Extraction of Road Junction Islands from High Resolution Aerial Imagery Using Level Sets, The International Archives of the Photogrammetry, Remote Sensing and Spatial Information Sciences. Vol. XXXVII. Part B3a. Beijing 2008

[17] Bibo Lu, Yongxia Ku, Hui Wang, "Automatic Road Extraction Method Based on Level Set and Shape Analysis," icicta, vol. 3, pp.511-514, 2009 Second International Conference on Intelligent Computation Technology and Automation, 2009

[18] Qihui Zhu, Mordohai, P.; , "A minimum cover approach for extracting the road network from airborne LIDAR data," Computer Vision Workshops (ICCV Workshops), 2009 IEEE 12th International Conference on, vol., no., pp.1582-1589, Sept. 27 2009-Oct. 42009

[19] A.Grote , M. Butenuth, C. Heipke,Road Extraction in Suburban Areas Based on Normalized Cuts PIA07 Photogrammetric Image Analysis --- Munich, Germany, September 19-21, 2007

[20] Anne Grote, Franz Rottensteiner,Automatic Road Network Extraction in Suburban Areas from Highresolution Aerial Images: Paparoditis N., PierrotDeseilligny M., Mallet C., Tournaire O. (Eds), IAPRS, Vol. XXXVIII, Part 3A - Saint-Mandé, France, September 1-3, 2010

[21] Luminita A.Vese and Tony F.Chan,( 2001) A multiphase level set framework for image segmentation using the Mumford and Shah model, UCLA CAM Report ,(2001) $01-25$

[22] Chunming Li , Chenyang Xu , Changfeng Gui , and Martin D. Fox (2005), Level Set Evolution Without Reinitialization: A New Variational Formulation Proceedings of the 2005 IEEE Computer Society Conference on Computer Vision and Pattern Recognition (CVPR'05) 2005

[23] Shi, J. and Malik J. (2000) Normalized cuts and image segmentation. IEEE Transactions on Pattern Analysis and Machine Intelligence 22(8): 2000 888-905.

[24] Wiedemann. C, Heipke. C, Mayer. H and Jamet. O (1998). Empirical evaluation of automatically extracted road axes. Empirical Evaluation Methods in Computer Vision. IEEE Computer Society Press. 1998 pp. 172187. 